\title{
Physical education: Preventative health care for youths
}

\author{
Alexandra Stoddart
}

\section{University of Western Ontario}

Obesity is at an epidemic level in this country. In recent years, the trend towards obesity has increased with 26 percent of women overweight in 2000, compared to 14 percent in $1985 .{ }^{1}$ Forty percent of men were overweight in 2000, upwards from 22 percent in $1985 .{ }^{1}$. Physical inactivity is also costing Canada massive amounts of money; in 2000 it was reported that about $\$ 2.1$ billion of the total health care costs in Canada were attributable to physical inactivity. ${ }^{2}$ Seventy percent of adolescents with obesity will grow up to become obese adults, and they will have more severe obesity than those who become obese in adulthood. ${ }^{3}$ With the notable increases in both obesity and diabetes, physical education (PE) should be strongly advocated for youths in order to diminish these obesity trends.

Current Canadian physical activity guidelines recommend that children accumulate a daily minimum of 60 minutes of moderate to vigorous physical activity (PA). ${ }^{4}$ Additionally, it is recommended that all children have at least 150 minutes of curricular instruction in PE per week..$^{5}$ This is wherein the problem lies. While some provinces have introduced mandatory daily PA, no province has educational policies mandating daily $P E$, and $P E$ is rarely a requirement for grades $10-12 .{ }^{5}$ In many cases, $\mathrm{PE}$ is the only opportunity for children to be active throughout the day.

Various associations, such as the Canadian Medical Association, recommend daily, quality PE in kindergarten through grade 12 because physical inactivity is such a strong contributor to being overweight. ${ }^{5}$ Reducing sedentary behaviours to less than 2 hours a day is critical for increasing PA and for increasing good health6. There is extensive evidence that PA decreases the risk of cardiovascular disease, regulates blood pressure, lowers the risk of certain cancers and diabetes, and is beneficial for arthritis. ${ }^{2,5,7}$ PA has beneficial mental health effects as well. Current research suggests that bouts of PA facilitate children's performance on tests that measure attention, memory, rapid decision making, and planning. ${ }^{8}$ Simultaneously, low aerobic fitness and obesity in children are associated with poorer academic performance. ${ }^{8}$ Research has demonstrated that high quality PE programs result in vast improvements in physical fitness levels and an enhanced academic performance.

A high quality PE program allows students to be active for the entirety of the class, build a fundamental skill base, and participate in interactive ways. Unlike the old gym class that was focused on team sports and dodge ball, now children can learn multiple ways to exercise that will be relevant throughout their lifetime. For schools that have the funds, the use of heart rate monitors can help students become aware of how their bodies respond to exercise. ${ }^{9}$ Interactive games like Dance Dance Revolution have been successful in raising daily PA levels, as well as increasing exercise motivation and adherence. ${ }^{7}$ With these types of exergaming programs, individuals are more likely to exercise for fun instead of focusing on losing weight. ${ }^{7}$ As children age, this will become especially critical as enjoyment remains one of the main factors of adherence. ${ }^{7}$ Health report cards have also been used as an aid in obesity prevention. Parents who received health and fitness reports were almost twice as likely to acknowledge that their child was actually overweight compared to those who did not get a report card. ${ }^{3}$ Additionally, the parents who received the health report cards were over twice as likely to plan weight control activities for their children. ${ }^{3}$ In addition to changes within the PE curriculum, PA can be integrated into academic subjects. ${ }^{10}$ Using a multidisciplinary approach (e.g. combining geography and PE), students can be active for a greater portion of the day. ${ }^{10}$ Aside from the health benefits that daily PA provides, it also contributes to instilling lifelong healthy values. By introducing PA early in life, it allows children to value and live an active lifestyle instead of a sedentary one, thus altering the obesity trends evident today. 
At the most basic level, physical education is an active form of health care. Rapidly growing literature continues to link physical inactivity with obesity and diabetes. Prevalence of obesity in children is increasing at a rapid rate. Children should be the prime group targeted for obesity intervention strategies because almost all children are accessible in schools. ${ }^{5}$ For those who are making decisions about education curriculum at the present time, it should be clear that physical education is a vital component of all schooling, regardless of age. In order to change the rising trend of obesity, Canadians need to be proactive instead of reactive. Obesity is a problem that will not simply go away; physical education has the potential to positively impact this predicament.

\section{References}

1. Canadian Fitness and Lifestyle Research Institute. FAQs. http://72.10.49.94/ node/2. Accessed March 102012.

2. Katzmarzyk T, Gledhill N, Shephard R. The economic burden of physical inactivity in Canada. CMAJ. 2000;163(11):1435-1440.

3. Dehghan M, Akhtar-Danesh N, Merchant A. Childhood obesity, prevalence and prevention. Nutrition Journal. 2005; 4:24.

4. Tremblay M, Warburton, D, Janssen I, Paterson, D, et al. New Canadian physical activity guidelines. Applied Physiology, Nutrition, and Metabolism. 2011;36(1):36-46.

5. CAHPERD. The need for quality physical education programs in Canadian schools.http://www.nationalchildrensalliance.com/nca/pubs/2006/ Quality\%20Physical\%20Education\%20Programs\%20Policy\%20Brief.pdf. Accessed March 272012.

6. Strong et al. Evidence based physical activity for school-age youth. The Journal of Pediatrics. 2005;146(6): 732-737.

7. Leininger LJ, Coles M, Gilbert J. Comparing enjoyment and perceived exertion between equivalent bouts of physically interactive video gaming and treadmill walking. Health and Fitness Journal of Canada. 2010;3(1):1218.

8. Tomporowski P, Lambourne K, Okumura M. Physical activity interventions and children's mental function: an introduction and overview. Preventive Medicine. 2011;52(Suppl 1):S3-S9.

9. Chorney D, \& Gunn, T. Utilizing heart rate monitors in today's physical education classroom. CAPHERD. 2009;71(4):18-21.

10. Wechsler H, McKenna M, Lee S, Dietz W. The role of schools in preventing childhood obesity. The State Education Standard. 2004:4-12.

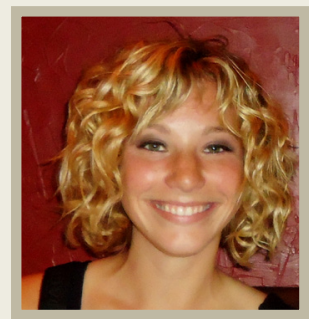

Alexandra Stoddart

Alexandra Stoddart is currently pursuing her MA in Kinesiology at the University of Western Ontario. She received her BSc and PE teaching certification from Eastern Michigan University. Her main research interests include motor learning and cognitive inhibition. 